\title{
Contexto
}

\section{La investigación contable colombiana frente a los nuevos criterios de medición de Colciencias}

\section{Editorial}

Hugo A. Macías*

Desde 2013, la profesora Yuliana Gómez Zapata ha liderado un interesante proceso en el Tecnológico de Antioquia, que condujo a la creación de la revista EnContexto, a su temprana indexación en Colciencias (finales de 2015), a publicar dos números por año desde este volumen 4 y a avanzar rápidamente hacia esferas más altas de cualificación. Esta revista es un ejemplo claro de que se pueden tener iniciativas desde la Contaduría Pública, con impacto en las ciencias económicas en general, que rápidamente sean avaladas por los criterios nacionales en materia de ciencia y tecnología. El apoyo institucional de las directivas del Tecnológico de Antioquia, en particular del Dr. Lorenzo Portocarrero Sierra desde la rectoría, ha sido clave para que la revista pueda avanzar rápidamente, ajustándose a los criterios nacionales e internacionales de calidad.

Más allá del ejercicio de la escritura en facultades de ciencias económicas, al que hicieron referencia las primeras páginas del primer número (Gómez, 2013) y teniendo en cuenta que En-Contexto es una publicación que nació como “(...) una alternativa para sentir, discutir y disentir en torno a los más variados temas de nuestro campo de conocimiento social" (Sarmiento, 2014, p.21). En esta editorial se pretende hacer unas recomendaciones específicas frente a los retos que ha puesto recientemente Colciencias a los grupos,

* Economista y MSc. en Economía, Ph.D. (c) en Administración. Profesor asociado al Programa de Contaduría Pública de la Universidad de Medellín y miembro de la Red Colombiana de Editores y Revistas Contables REDITORES, Medellín - Colombia.

hmacias@udem.edu.co 


\section{Conterto mecass}

revistas e investigadores contables en Colombia. La contribución de este documento está en los últimos cuatro párrafos, pero ellos están soportados en la discusión que se presenta a continuación.

Si bien hace más de 10 años que los académicos contables se vienen adaptando a los lineamientos de Colciencias, recientemente han aparecido nuevos criterios de medición para el desempeño de grupos, revistas e investigadores. A los grupos se les clasifica en A1, A2, B, C, D o reconocido; las revistas se clasifican en A1, A2, $\mathrm{B}$ o C; los investigadores se clasifican en senior, asociado o junior. Lo nuevo de los criterios de medición -a lo que se hará referencia aquí- es que tanto los grupos, como las revistas y los investigadores tendrán que tener más vínculos con las revistas que hacen parte de Web of Science (anterior ISI) y de Scopus.

Con respecto a los grupos, el modelo de medición presentado por Colciencias en diciembre de 2015, contiene productos top, tipo A, tipo B, indicadores de producción, de cohesión, de cooperación, umbrales por área del conocimiento, entre otros (Colciencias, 2015). Este modelo de medición es bastante complejo por sus especificidades técnicas y amerita un análisis extenso y profundo al interior de cada grupo de investigación contable. Aquí se hará referencia exclusivamente al reto de publicar en revistas que estén en los cuartiles Q1, Q2, Q3 y Q4 de Web of Sciece y de Scopus.

La clasificación de cada revista en Q1, Q2, Q3 o Q4 se define anualmente a partir del numero de citas que cada revista ha recibido; esa clasificación se publica como Journal Citation Reports (JCR) para las revistas de Web of Science y como SCImago Journal Rank (SJR) para las publicaciones de Scopus. El procedimiento consiste en listar las revistas por orden de citación y dividir la lista en cuatro grupos, de manera que el grupo más citado es el cuartil Q1 y el cuartil menos citado es Q4. El SJR 2015 tiene 126 revistas contables, donde las 32 revistas mas citadas son Q1, el grupo de 31 revistas que sigue en citación está en Q2, las 31 que siguen son Q3 y las 32 publicaciones con menor número de citas, son Q4. Por su parte, el JCR 2015 incluye solo 25 revistas contables, distribuidas en los cuatro cuartiles.

A través del modelo de medición, Colciencias le está pidiendo a los grupos de investigación contable que participen activamente en la comunidad que discute en estas 126 revistas. De esas 126 publicaciones, 80 tienen la palabra "accounting" en su denominación, las otras 46 son revistas que publican documentos contables, pero que no están especializadas en contabilidad. En esas 80 revistas contables que aparecen en Scopus no hay ningún artículo que provenga de alguno de los 62 grupos de investigación contable escalafonados por Colciencias el 20 de mayo de 2016. Eso no pasa en otros países de la 
región, como en México, donde los académicos contables tienen más de 20 artículos publicados en esas 80 revistas, en Brasil donde han publicado más de 40 artículos allí, ni en otros países más pequeños que también tienen una participación activa (Macías, 2013a).

Es importante no caer en valorar solo los resultados en su fase final, convirtiendo los productos JCR y SJR en fetiches de indicadores cuantitativos, sino asegurarse de que cada producto tenga detrás una estructura robusta, con procesos investigativos sólidos e importantes avances disciplinares (Machado, Patiño y Cadavid, 2016, pp.56-57). Si bien existe una tradición de varias décadas de investigación contable en Colombia alrededor de la Federación Nacional de Estudiantes de Contaduría Pública, FENECOP, (Sarmiento y Muñoz, 2011) y el CCINCO (Centro Colombiano de Investigación Contable) también ha trabajado varias décadas para desarrollar la investigación contable (Barrios, Fúquene y Lemos, 2010), el número de grupos reconocidos por Colciencias ha crecido solo recientemente. En 2004 solo un grupo contable estaba reconocido por Colciencias, en 200611 grupos lograron escalafón, en 2008 pasaron a 33 (Macías y Cortés, 2009, pp.28-30) y han venido creciendo hasta llegar a los 62 grupos con escalafón vigente en 2016. Las características de esos grupos ya han sido analizadas formalmente, en términos de líneas de investigación, publicaciones, proyectos, entre otros (Valero y Patiño, 2012), lo cual permite identificar sus retos y hacer una propuesta de mejoramiento basada en los nuevos lineamientos de Colciencias. Las recomendaciones para los grupos se presentan al final de este documento.

Para el caso de las revistas, las nuevas exigencias de Colciencias son mucho más fuertes, en el sentido de que los vínculos con Web of Science y con Scopus son más exigentes. A finales de mayo de 2016 Colciencias publicó una propuesta de "política para mejorar la calidad de las publicaciones científicas nacionales", con dos objetivos: (1) aumentar la participación de los investigadores nacionales en revistas JCR y SJR, así como (2) aumentar la participación de las revistas científicas nacionales en estas mismas bases de datos (Colciencias, 2016). El nuevo modelo de clasificación propuesto implica cambios radicales, ya que las revistas serían A1 solo si están en los cuartiles Q1 y Q2 de JCR o SJR y para llegar a A2 habría que estar en los cuartiles Q3 y Q4 de JCR o SJR; la clasificación en $\mathrm{B}$ y $\mathrm{C}$ dependerá de cuartiles construidos para cada gran área del conocimiento, con base en las citas registradas en Google Académico.

En 2016 hay más de 50 revistas de "Economía y Negocios" indexadas en el Publindex de Colciencias, de las cuales solo 7 están en Scopus, 6 en Q4 y 1 en Q3. Ninguna de estas 7 revistas es contable y ninguna de ellas está en JCR. Para cumplir el primer objetivo de la nueva política, Colciencias propuso: (1) 


\section{Conterto mecass}

capacitar a los autores en elaboración de artículos de calidad y en selección de revistas para publicar y (2) fomentar la participación de pares internacionales en los proyectos. Para cumplir el segundo objetivo, propuso: (1) rediseñar el modelo de clasificación de revistas nacionales; (2) revisar permanentemente los criterios, con acompañamiento de editores; (3) actualizar constantemente a la comunidad científica nacional en bibliometría y cienciometría; (4) actualizar constantemente a los equipos editoriales de las revistas; (5) renovar el portal de Publindex con cursos virtuales y (6) apoyar, desde Colciencias, las revistas para que cuenten con la infraestructura necesaria (Colciencias, 2016, pp.15-16).

En los últimos 5 anos, en Colombia se han dado importantes discusiones sobre distintos aspectos de las revistas contables. No solo se han analizado las trayectorias de algunas revistas nacionales y sus niveles de cualificación (Santos, 2012; Méndez, 2013; Macías y Patiño, 2014) y el incipiente nivel de desarrollo de las revistas contables latinoamericanas (Macías y Moncada, 2011; Ayala, 2014), sino que se consideran una plataforma para la construcción de conocimiento contable (Macías, 2012) y se llevó a cabo un debate escrito sobre el futuro de las revistas contables colombianas, que en su cuarta versión contó con la participación de editores de 14 revistas (Alzate, et al., 2014). La principal respuesta a estas discusiones fue la conformación de la Red Colombiana de Editores y Revistas Contables REDITORES en el primer semestre de 2015 (Castaño, 2015; Rueda, 2015), desde donde se espera construir una interacción formal con Colciencias en estos procesos de clasificación de revistas.

En el lanzamiento de REDITORES, el profesor Fredy León Paime presentó un decálogo de recomendaciones para los editores, que ha sido reproducido en varias revistas de la Red. Los diez puntos son: (1) todo lo que se haga para construir comunidad científica es necesario; (2) tenemos hoy mucha publicación y poca diferenciación; (3) las revistas no deben competir, sino generar redes de colaboración temática y metodológica; (4) las revistas no son propiedad exclusiva de las instituciones que las producen, ni de sus editores, tienen una función particular dentro de un campo colectivo interinstitucional; (5) la comunidad, más que las instituciones editoras, es responsable de generar el camino de transición hacia los nuevos requerimientos nacionales, para que no se pierdan los capitales culturales constituidos; (6) no hay que temer al tribalismo, a valorar la diferencia y el disenso, en esta etapa de las revistas contables; (7) los editores no están solos, pueden seguir madurando los acuerdos cooperativos existentes; (8) hay que luchar por mantener la autonomía, en medio de todas estas exigencias externas; (9) la experiencia de editor es apasionante, pero también transitoria y (10) se necesita que los editores sean creativos, que mantengan un compromiso propositivo y que sean conscientes del rol central que juegan en el campo (León-Paime, 2015). Al final de este texto se presentan unas recomendaciones para las revistas contables 
a partir de este decálogo, de las discusiones acumuladas y de los nuevos criterios de clasificación de Colciencias.

Como si fuera poco hacer seguimiento sistemático a los grupos y las revistas, Colciencias ahora está midiendo y escalafonando también a los investigadores. En el documento publicado en diciembre de 2015 también está incluido el modelo de "reconocimiento de investigadores", que los clasifica en senior, asociado y junior, según el nivel de formación, la producción académica y los productos de formación. Para que un investigador contable llegue a ser senior, debe tener título de doctorado, 10 artículos publicados en los últimos 10 años en revistas JCR o SJR que estén en Q1 o Q2 y haber dirigido cuatro trabajos de maestría o una tesis doctoral terminada en los últimos 10 años (Colciencias, 2015). El modelo realmente es flexible y define otros productos equivalentes, pero estos datos dan una idea del nivel de exigencia y de la necesidad que tiene la academia contable de enfrentar estas nuevas demandas.

Para ir avanzando en las categorías de investigador hay que irse incorporando en los hábitos del investigador y desarrollar respuestas personales a estas demandas. En el trabajo de Macías y Cortés (2009, p.27) se planteó incluso el concepto de "disposición escolástica" como punto de referencia, que fue retomado en el trabajo de Machado, Patiño y Cadavid (2016, pp.52-53). Se plantea allí que además de dedicar tiempo a la investigación, es necesaria una disposición para ella, un gusto, una pasión, que la investigación sea tomada como una opción de vida. Eso implica conocer la comunidad académica que trabaja sobre cada línea de investigación abordada por un autor, reconocer los trabajos previos con sus avances y vacíos, para entrar en diálogo directo y escrito con esa comunidad.

Un estudio publicado en 2010 identificó las principales debilidades de los académicos contables colombianos: (1) la mayoría no dominan idiomas distintos al español; (2) baja formación en posgrados contables; (3) casi la mitad de los investigadores no tiene experiencia empresarial; (4) el tiempo dedicado a la investigación oscila entre 6 y 10 horas semanales y (5) muy escasa producción de nuevo conocimiento (Patiño, Romero y Jara, 2010). En ese mismo trabajo se insistió en la necesidad de un mayor número de académicos formados en maestrías y doctorados contables, capacitación en idiomas y propiciar un aumento sustancial del tiempo real dedicado a investigación.

El hecho de que la investigación contable colombiana tenga "falencias estructurales" y dentro de ellas "carencia de investigadores formados para investigar" (Machado, et al., 2016, p.63), se ha enfrentado de distintas maneras. En algunos casos se hacen por ejemplo esfuerzos de "alfabetización académica" 


\section{Conterto mecass}

de estudiantes de los primeros semestres (Ruíz y Muñoz, 2015), se desarrollan experiencias didácticas y pedagógicas que conduzcan a escribir en un ambiente de no lectores (Grajales, 2010) y también se desarrollan semilleros formales de investigación contable a lo largo del territorio nacional (Hoyos, Rocha, Duque, Rodríguez, Gómez y Leiva, 2014). Por fuera del currículo se desarrollan importantes procesos en el marco de FENECOP (Sarmiento y Muñoz, 2011) y otros congresos anuales de estudiantes que llevan más de una década. Como complemento, se está incrementando el número de estudiantes de todo el país en las maestrías contables de Bogotá, Cali y Medellín (algunas con extensión a otras ciudades) y un número pequeño de profesores se ha estado formando muy recientemente en doctorados europeos en contabilidad.

El trabajo de los grupos, las revistas, los investigadores y su vinculación con JCR y SJR, converge en la estructura de los artículos. Es allí donde se refleja la formación de los autores, la literatura que estudian, las discusiones en las que participan, los esfuerzos institucionales y personales para la ejecución de proyectos rigurosos, la cooperación entre académicos y la distancia con las prácticas profesionales al interior de las empresas. Es allí donde se refleja la vinculación con la corriente interpretativa (Rueda, 2007; Macías, 2013b) o con la corriente crítica (Macías, 2016) y el desarrollo de trabajo de campo como complemento necesario a las revisiones documentales (Alzate J. S., 2014). La estructura de los artículos debe ubicarse en el primer nivel de atención de los académicos contables, no solo porque Colciencias ya detectó que es ahí donde se debe trabajar, sino porque es ahí donde los académicos le dan sentido al trabajo que se hace.

Con respecto a los artículos, no se trata de escribir por escribir, de escribir para sumar puntos salariales o para poner el "chulito" en el formato que envía el directivo universitario. Tampoco se trata de escribir bonito, de sorprender con las palabras, de presentar un discurso denso que pocos entienden. Lo importante es posicionarse en la literatura previa sobre lo que se está abordando, para destacar el mensaje central, lo novedoso, el aporte, la contribución. Ese aporte no tiene que ser definitivo, no tiene que ser terminal, pero sí debe ser diferenciable de lo que se ha escrito previamente, para que pueda dársele continuidad más adelante en otro texto del mismo autor o de otros autores. Es necesario "no redundar en lo ya trabajado" (Machado, et al., 2016, p.49). Cada artículo publicado en una revista con pretensiones científicas debe contribuir de manera clara y explícita a la construcción colectiva de conocimiento; para otro tipo de textos hay otros espacios.

Luego de presentar los nuevos parámetros de Colciencias y las discusiones que se han dado sobre esos temas en la academia contable colombiana, se procede a hacer recomendaciones específicas para revistas, investigadores y grupos. Para incrementar el nivel de citación de las revistas colombianas con importantes 
discusiones contables, se hacen cinco recomendaciones concretas. (1) Declarar la identidad de cada revista con respecto a las demás, en términos de enfoque, principales temas de discusión y preferencias metodológicas. (2) Exigir a los autores nacionales y extranjeros que retomen las discusiones nacionales sobre los temas y los métodos que abordan; cada artículo debe contextualizarse en las discusiones nacionales, para que su contribución sea explícita y clara, con respecto a lo que ya está publicado en la academia contable nacional. (3) Publicar ediciones especiales de las revistas, sobre temas definidos colectivamente, que identifiquen a cada revista como fuente importante de una línea o una perspectiva particular. (4) Invitar a académicos contables ya posicionados, con alto nivel de citación, a ser parte de los comités de las revistas y a participar como autores en las mismas. (5) Desarrollar actividades orientadas a incrementar el nivel de citación de las revistas colombianas con discusiones contables, desde la Red colombiana de editores y revistas contables, REDITORES.

Para ascender en la categoría de investigador, se hacen cinco recomendaciones específicas. (1) Mantener al menos un proyecto de investigación formal por año, aprobado por alguna institución de educación superior; los proyectos formales son la fuente natural de todos los productos de investigación. (2) Construir un plan personal, por parte de cada investigador, para empezar a tener al menos una publicación cada dos años en revistas contables JCR o SJR; esto implica estudiar sistemáticamente las revistas de estas bases donde se discute la línea de investigación de interés, para identificar tanto las contribuciones potenciales, como las alianzas, relaciones y recursos necesarios para lograr entrar en esas discusiones; se recomienda identificar las convocatorias para números especiales y trabajar con nuevos coautores. (3) Participar como ponente en los eventos nacionales y en los principales congresos internacionales (particularmente aquellos donde hacen presencia explícita los journal contables), para fortalecer las relaciones directas con los demás interesados en la misma línea de investigación. (4) Dirigir al menos dos trabajos de grado por año, de pregrado o maestría, donde sea clara la orientación contable, la audiencia a la que están dirigidos y la contribución; si cada trabajo de grado hace una contribución en el contexto colombiano, cada trabajo puede conducir al menos a una publicación en una revista nacional. (5) Incorporar trabajo empírico en cada investigación, ir más allá de las revisiones documentales.

Para los grupos también se hacen cinco recomendaciones. (1) Superar la dependencia de los capítulos y libros, para el fortalecimiento de los grupos de investigación; la baja circulación de los libros y las exigencias de citación, hacen que una estrategia basada en libros no sea sostenible en el tiempo. (2) Construir una estrategia de mediano plazo para participar en las discusiones internacionales de los journal, basada en el conocimiento preciso de las 


\section{Conterto mecass}

publicaciones donde se discuten las líneas de investigación del grupo, con enfoque metodológico similar, para propiciar interacción de varios integrantes del grupo con autores de esos espacios. (3) Diseñar estrategias para acceder a recursos de Colciencias, ICETEX, de gobiernos y otros organismos nacionales e internacionales, destinados a proyectos, formación de investigadores y movilidad internacional. (4) Desarrollar investigaciones empíricas y productos que tengan relación directa con las necesidades de las organizaciones y de la profesión, en el contexto colombiano. (5) Construir y ejecutar un plan de capacitación de los integrantes del grupo, para enfrentar estos retos que aún son poco familiares para la mayoría de los académicos contables.

El principal reto para la academia contable colombiana es comprender la dinámica de la academia contable anglosajona, esa que es medida por el JCR y el SJR, para poder incorporarse paulatinamente en esas discusiones. Un número muy pequeño de académicos contables ya lo está haciendo, pero es necesario incrementar sustancialmente esta dinámica, como lo han hecho de tiempo atrás los académicos colombianos de economía y de administración. El reto principal para la academia contable es integrarse a las discusiones anglosajonas, tanto desde la formación, como la publicación de artículos y la interacción formal de las revistas contables colombianas con las extranjeras.

\section{Referencias}

Alzate, J. (2014). La investigación contable pensada desde las estrategias de investigación social-cualitativa. Teuken Bidikay, 5, 77-97.

Alzate, J., Bautista, J., Cano, A., Castaño, C., Ceballos, R., Cortés, J.,... Sarmiento, H. (2014). El futuro de las revistas contables colombianas: un debate polifónico. Cuadernos de Contabilidad, 15(37), 261-278.

Ayala, J. (2014). Producción contable en universidades afiliadas a la Asociación Latinoamericana de Facultades y Escuelas de Contaduría y Administración (ALAFEC): Caso Argentina, Chile y México. Criterio Libre, 12(21), 221-248.

Barrios, C., Fúquene, T., y Lemos, J. (2010). Desarrollo de la investigación contable en el centro colombiano de investigación contable. Contaduría y Administración, 231, 151-177.

Castaño, C. (2015). El nacimiento de la Red Colombiana de Editores y Revistas Contables. Contaduría Universidad de Antioquia, 67, 11-12.

Colciencias. (2015). Modelo de medición de grupos de investigación, desarrollo tecnológico o de innovacion y de reconocimiento de investigadores 
del sistema nacional de ciencia, tecnología e innovacion. Recuperado de http://www.colciencias.gov.co/sites/default/files/upload/noticias/ mediciondegrupos-actene2015.pdf

Colciencias. (2016). Política para mejorar la calidad de las publicaciones cientificas nacionales. Versión para discusión. Recuperado de http:// www.colciencias.gov.co/sites/default/files/upload/paginas/politicapublindex-colciencias.pdf

Gómez, Y. (2013). El ejercicio de la escritura: un reto para las facultades de ciencias económicas en Colombia. En-Contexto, 1, 7-10.

Grajales, J. (2010). La escritura de anteproyectos de investigación en un ambiente de no lectores. El caso del curso de metodología de la investigación contable en la Universidad del Valle Sede Norte del Cauca. Contaduría Universidad de Antioquia, 57, 145-159.

Hoyos, M, Rocha, M., Duque, O., Rodríguez, J., Gómez, S. y Leiva, P. (2014). Caracterización de los semilleros de investigación en los programas de Contaduría Pública en Colombia. Activos, 23, 133-180.

León-Paime, E. (2015). Carta al editor contable. Visión Contable, 13, 11-14.

Machado, M., Patiño, R. y Cadavid, Y. (2016). La investigación contable en Colombia. En M. Machado. (Ed.), Caminos Contables: Problemas y metodologías para el desarrollo de la investigación (pp.49-85). Medellín, Colombia: Sello Editorial Centro de Investigaciones y Consultoruas -CIC-.

Macías, H. (2012). Las revistas académicas como plataforma para la construcción de conocimiento contable. Revista Facultad de Ciencias Económicas: Investigación y Reflexión, XX(2), 5-17.

Macías, H. (2013a). Internacionalización de las revistas contables: un aporte al debate polifónico. Cuadernos de Contabilidad, 14(35), 785-794.

Macías, H. (2013b). Vínculos de la investigación contable interpretativa con la producción académica colombiana: avances y oportunidades. Cuadernos de Contabilidad, 14(35), 699-727.

Macías, H. (2016). Introducción a la investigación contable crítica (ICC) en su contexto original. En Z. Melgarejo, y M. Vera. (Ed.), Investigación global en contabilidad y finanzas. Contribuciones a la perspectiva crítica en contabilidad, finanzas y gestión (p. en prensa). Bogotá, Colombia: Universidad Nacional de Colombia.

Macías, H. y Cortés, J. (2009). El campo de la investigación contable: oportunidades para los investigadores colombianos. Cuadernos de Contabilidad, 10(26), 21-50. 


\section{Conterto mecass}

Macías, H. y Moncada, T. (2011). El campo científico de la contabilidad: panorama internacional de las revistas. Revista Lúmina, 12, 260-278.

Macías, H. y Patiño, R. (2014). Evolución de las revistas contables colombianas: de la reflexión a la investigación. Contaduría Universidad de Antioquia, 64, 13-48.

Méndez, K. (2013). La indexación de las revistas contables en Colombia. Activos, 21, 171-190.

Patiño, R., Romero, A. y Jara, K. (2010). Características de los investigadores relacionados con programas de Contaduría Pública y con temáticas relacionadas. Cuadernos de Contabilidad, 11(28), 171-199.

Rueda, G. (2007). La investigación contable: vínculos ontológicos y las posibilidades de la investigación interpretativa. Contaduría Universidad de Antioquia, 50, 119-132.

Rueda, G. (2015). Red colombiana de editores y revistas contables, REDITORES. Cuadernos de Contabilidad, 16(41), 245-247.

Ruíz, G. y Muñoz, S. (2015). Experiencia didáctica de alfabetizacion académica para estudiantes de primer semestre de Contaduría Pública. Visión Contable, 13, 30-51.

Santos, G. (2012). Aproximaciones a un análisis bibliométrico de la Revista Internacional Legis de Contabilidad \& Auditoría 2000-2012. Revista Internacional Legis de Contabilidad \& Auditoría, 15(49), 183-226.

Sarmiento, H. (2014). Una segunda oportunidad. En-Contexto, 2, 21-24.

Sarmiento, H. y Muñoz, S. (2011). Banderas en alto. Una arqueología del proceso de nacionalización de la Contaduría Pública en Colombia. Medellín, Colombia: Politécnico Colombiano Jaime Isaza Cadavid.

Valero, G. y Patiño, R. (2012). Los grupos de investigación contable reconocidos por Colciencias. Cuadernos de Contabilidad, 13(32), 175-201.

\section{Para citar este artículo:}

Macías, H. (2016). La investigación contable colombiana frente a los nuevos criterios de medición de Colciencias. En-Contexto, 4(4), 39-48 